\title{
Serum miR-4530 sensitizes breast cancer to neoadjuvant chemotherapy by suppressing RUNX2
}

This article was published in the following Dove Press journal: Cancer Management and Research

\author{
Xiao-Xiao Wang ${ }^{1, *}$ \\ Fu-Gui Yer,* \\ Jie Zhangl,* \\ Jun-Jing $\mathrm{Li}^{\prime}$ \\ Qing-Xia Chen' \\ Pei-Yang Lin' \\ Chuan-Gui Song'
}

'Department of Breast Surgery, Affiliated Union Hospital, Fujian Medical University, Fuzhou, People's Republic of China; ${ }^{2}$ Department of Breast Surgery, Key Laboratory of Breast Cancer, Fudan University Shanghai Cancer Center, Shanghai Medical College, Fudan University, Shanghai, People's Republic of China

*These authors contributed equally to this work
Correspondence: Chuan-Gui Song Department of Breast Surgery, Affiliated Union Hospital, Fujian Medical University, 29 Xin-Quan Road, Fuzhou 35000I, People's Republic of China Tel +86 I 3960709993

Email songchuangui@yahoo.com
Purpose: Neoadjuvant chemotherapy (NAC) plays a pivotal role in the treatment of locally advanced breast cancer (LABC); however, breast cancer is a heterogeneous disease, individual responses to chemotherapy are highly variable. Therefore, the purpose of the current research is to identify biomarkers that can predict the chemotherapeutic response.

Patients and methods: We recruited 78 patients with primary breast cancer who underwent taxane- and anthracycline-based NAC; these patients were divided into sensitive and resistant groups according to the Response Evaluation Criteria in Solid Tumors (RECIST) criteria. The microRNA microarray was conducted to explore differentially expressed miRNAs. Quantitative real-time polymerase chain reaction (qRT-PCR) further validated the relationship between miR-4530 and chemosensitivity in breast cancer patients.

Results: No significant differences were observed between the two groups regarding the clinicopathological characteristics. miR-4530 showed the most potential involving breast cancer chemosensitivity. Mechanically, RUNX2 was identified one of the direct targets of miR-4530 and responsible for breast cancer chemosensitivity.

Conclusion: Our results revealed that elevated serum miR-4530 levels may sensitize breast cancer to taxane- and anthracycline-based NAC by suppressing RUNX2; therefore, this miRNA has the potential to be a new biomarker for predicting breast cancer chemosensitivity.

Keywords: miR-4530, serum, neoadjuvant chemotherapy, chemotherapeutic resistance, RUNX2

\section{Introduction}

Breast cancer remains one of leading causes of cancer-related mortality in women worldwide. ${ }^{1}$ In China, the incidence of breast cancer continues to increase, with patients diagnosed with locally advanced breast cancer (LABC) comprising a significantly high percentage of all cases. Neoadjuvant chemotherapy (NAC) plays a pivotal role in the treatment of LABC and has been shown to downstage lesions and increase the incidence of breast-conserving surgery (BCS). ${ }^{2-4}$ However, breast cancer is considered a heterogeneous disease such that chemotherapy sensitivity varies with the individual, and chemotherapy resistance frequently culminates in subsequent breast cancer recurrence and metastasis. To date, there are no validated, effective, and precise biomarkers available to predict a patient's chemotherapeutic response. Therefore, identifying patients who might benefit from NAC prior to treatment initiation is still urgently needed.

microRNAs (miRNAs) are non-coding, single-stranded RNAs 20-25 nucleotides in length that posttranscriptionally regulate gene expression by either degrading the target mRNA or inhibiting its translation. It has been revealed that miRNAs that act as 
either oncogenes or tumor suppressors are virtually involved in many biological processes such as cell proliferation, differentiation, apoptosis, metabolism, adhesion, signal transduction, and cell cycle regulation..$^{5-7}$ Over the past decade, increasing attention has been paid to circulating miRNAs due to their high stability and potential as biomarkers for tracking and predicting the chemotherapeutic response of patients with breast cancer. ${ }^{8-12}$ Additionally, miRNAs can avoid RNase degradation because they are primarily contained within lipoprotein vesicles and exosomes as well as bound to RNAbinding proteins such as Argonaut 2. ${ }^{13-15}$

In 2008, Lawrie et al ${ }^{16}$ first reported that serum levels of miR-21 were associated with relapse-free survival in patients with diffuse large B-cell lymphoma. Since then, accumulating evidence has revealed that circulating miRNAs are associated with chemotherapy resistance. When Zhao et al ${ }^{12}$ compared miR-221 expression levels in plasma, a significant difference was observed in overall response rate but not the pathological complete response ( $\mathrm{pCR}$ ) rate. Therefore, they concluded that plasma miR-221 could be a predictive biomarker for NAC sensitivity in breast cancer patients. Jung et $\mathrm{al}^{8}$ measured the expression levels of plasma miR-210 before and after NAC (including regimens containing trastuzumab) and found that circulating miR-210 levels were significantly higher in patients who had residual disease than in those who had achieved a pCR. Their results indicated that plasma miR210 levels could be used to predict and perhaps monitor the response of patients with HER2-positive breast cancer to an NAC with trastuzumab. In other studies, circulating miR125b, miR-19a, and miR-205 levels were also associated with chemotherapeutic resistance and serve as potential markers to predict the chemotherapeutic response. ${ }^{9,10}$

Our study was designed to compare the serum levels of miR-4530 between patients who had sensitive and resistant breast cancer prior to receiving NAC and to determine the association (if any) of relative miR-4530 expression levels with the patient's response to NAC. In addition, we aimed to explore the target genes of miR-4530 and its potential as a biomarker for predicting chemotherapeutic response.

\section{Materials and methods}

\section{Ethics statement}

This study protocol was approved by the ethical committee of the Affiliated Union Hospital of Fujian Medical University (Fuzhou, China), and written informed consent was obtained from all patients before the study was initiated. HS578T human breast cancer cell lines were obtained from the Shanghai Cell Bank Type Culture Collection Committee (CBTCCC,
Shanghai, China) in 2012 and cultured in complete growth medium as recommended by the distributor.

\section{Patients}

Between March 2014 and January 2016, we recruited 78 primary stage II-III breast cancer patients whose tumors were pathologically confirmed by core needle biopsy and who had received either six or eight cycles of taxane- and anthracycline-based NAC. The Response Evaluation Criteria in Solid Tumors (RECIST) criteria were used to assess the patients' response to NAC. Accordingly, 54 patients who achieved either a complete response (CR, disappearance of all target lesions) or partial response (PR, at least a 30\% decrease in the sum of diameters of target lesions) were stratified in the sensitive group, and 24 patients who experienced either disease stabilization (SD, neither sufficient shrinkage to qualify for PR nor sufficient increase to qualify for PD) or disease progression (PD, at least a $20 \%$ increase in the sum of the diameters of target lesions) were defined as the resistant group.

\section{Serum collection and RNA extraction}

Whole blood samples were collected at baseline (before NAC) and centrifuged at $1,600 \times g$ for 10 minutes within 2 hours of collection. The serum supernatant was transferred into centrifuge tubes and immediately stored at $-80^{\circ} \mathrm{C}$ until further use. Total RNA was extracted and purified by using the miRNeasy Serum/Plasma Kit (QIAGEN, Hilden, Germany) following the manufacturer's instructions. In addition, the concentration of the eluted RNA was quantified by using a NanoDrop ND-1000 spectrophotometer (Thermo Fisher Scientific, Waltham, MA, USA).

\section{Agilent human miRNA $\left(8^{*} 60 \mathrm{~K}\right)$ microarray}

For the initial screening phase, Agilent Human miRNA microarray 21.0 was used to identify profiles of differentially expressed candidate miRNAs from the serum samples and from the two groups (two samples from the sensitive group; two samples from the resistant group). This microarray was derived from the Sanger database version 21.0 and is capable of detecting 2,549 human microRNAs. Each slide was hybridized with $100 \mathrm{ng}$ of Cy3-labeled RNA using the miRNA Complete Labeling and Hyb Kit (Cat \# 5190-0456, Agilent Technologies, Santa Clara, CA, USA) according to the manufacturer's instructions and incubated in a hybridization oven (Cat \# G2545A, Agilent Technologies) at $55^{\circ} \mathrm{C}$ for 20 hours with a rotation of $20 \mathrm{rpm}$. Microarray slides were scanned 
with an Agilent Microarray Scanner (Cat \# G2565CA, Agilent Technologies). Raw data were normalized by using the Quantile normalization procedure in GeneSpring Software 12.6 (Agilent Technologies) and then log transformed with base 2 . Based on the results, we selected miR-4530 as the candidate miRNA.

\section{Quantitative real-time PCR (qRT-PCR)}

For the validation phase, we reverse transcribed total RNA by using the miScript Reverse Transcription Kit (QIAGEN) into a final volume of $20 \mu \mathrm{L}\left(60\right.$ minutes at $37^{\circ} \mathrm{C}, 5$ minutes at $95^{\circ} \mathrm{C}$, and maintained at $4^{\circ} \mathrm{C}$ ). Then, the synthesized cDNA samples were diluted with RNase-free water to $200 \mathrm{~mL}$ for subsequent qRT-PCR experiments that were conducted on an ABI StepOnePlus Real-Time PCR system using a miScript SYBR Green PCR Kit (QIAGEN) according to the manufacturer's instructions. All reactions were performed in duplicate with the following cycling conditions: an initial activation step at $95^{\circ} \mathrm{C}$ for 15 minutes and 40 cycles at $94^{\circ} \mathrm{C}$ for 15 seconds, $55^{\circ} \mathrm{C}$ for 30 seconds, and $70^{\circ} \mathrm{C}$ for 30 seconds. ce-miR-39 has been verified to be stably expressed in serum and thus was used as a normalization control for relative quantification of the miScript PCR System. The relative expression of miR-4530 in both groups was calculated from the following equation: fold change of relative $\mathrm{miR}-4530$ expression $=2^{-\Delta \mathrm{Ct}}$, where $\Delta \mathrm{Ct}$ is the mean $\mathrm{Ct}_{\text {miR-4530 }}$ minus the mean $\mathrm{Ct}_{\text {ce-miR-39. }}$.

\section{Reverse transcription PCR for evaluation of Runt-related transcription factor 2 (RUNX2) expression and dual luciferase reporter assay targeting RUNX2 3'-UTR}

We performed reverse transcription PCR using the PrimeScript RT reagent Kit with gDNA Eraser (Takara Bio Inc., Kyoto, Japan) to detect relative expression of RUNX2 in HS578T cells, which were co-transfected with the miR-4530 mimics or miR-control, according to the manufacturer's instructions.

Next, the 3'-UTR of RUNX2 was amplified from human genomic DNA using appropriate primers and cloned into the region directly downstream of the Renilla gene stop codon in the psiCHECK2 ${ }^{\mathrm{TM}}$ vector (Promega Corporation, Fitchburg, WI, USA). PCR amplification of the 3 '-UTR sequence of RUNX2 generated a series of mutant psiCHECK2-RUNX23'-UTR reporter vectors. The miR-4530 mimic duplexes were synthesized by Shanghai GeneChem Co. (Shanghai, China). Co-transfection with a mixture of reporter constructs and miR-4530 duplexes was performed in HEK293T cells using Lipofectamine 2000 (Invitrogen, Carlsbad, CA, USA).
After incubation for 48 hours, firefly and Renilla luciferase activities were measured using the Dual-Luciferase Reporter Assay system (Promega Corporation).

\section{Statistical analysis}

For microarray analysis, we conducted Student's $t$-tests to screen differentially expressed genes, and the Mann-Whitney $U$ test was performed to compare the serum miR-4530 levels. Differences in the clinical characteristics of the patients in the resistant and sensitive groups were estimated by the chisquared test. Area under the receiver operating characteristic (ROC) curves was used to assess the predictive accuracy of miR-4530. All statistical analyses were performed using SPSS 20.0 software (IBM Corporation, Armonk, NY, USA), and a two-sided $P$-value $<0.05$ was considered statistically significant.

\section{Results \\ Clinicopathological characteristics of the study population}

In the validation phase, a total of 74 patients met the eligibility criteria for our study. The RECIST criteria divided the entire cohort into two groups: a sensitive group comprising $52(70.27 \%)$ patients who responded to chemotherapy with either a PR or CR; and a resistant group comprising 22 (29.73\%) patients who experienced either SD or PD. Table 1 summarizes the clinicopathological characteristics of the study participants, which shows that no significant differences were observed in the characteristics between the two groups. The median ages of the two groups were 45 and 49 years old, respectively (range, 28-61 years). Regarding the cancer characteristics, a large percentage of patients were diagnosed with T2 tumors, and approximately three-fifths of the patients presented Luminal B subtype. Most of the patients did not achieve pCR, with pCR rates of $16.3 \%$ and $9.5 \%$ for the sensitive and resistant groups, respectively.

\section{Identification of differentially expressed miRNA profiles in the screening phase}

A microarray comprising probes targeting 2,549 human miRNAs was initially used to investigate significantly different expression levels of miRNAs between the two groups. When compared to the resistant group, we found 15 significantly upregulated miRNAs in the sensitive group; the complete list of differentially expressed miRNAs between the two groups is provided in Table $\mathrm{S} 1$. In addition, miR-4530 showed a 2.16-fold change between the sensitive and resistant groups. 
Table I Clinicopathological characteristics of patients in the sensitive and resistant groups

\begin{tabular}{|c|c|c|c|c|c|}
\hline \multirow[t]{2}{*}{ Characteristics } & \multicolumn{2}{|c|}{$\begin{array}{l}\text { Sensitive } \\
(n=52)\end{array}$} & \multicolumn{2}{|c|}{$\begin{array}{l}\text { Resistant } \\
(\mathrm{n}=22)\end{array}$} & \multirow[t]{2}{*}{$P$-value } \\
\hline & No & $\%$ & No & $\%$ & \\
\hline Age (year), median (range) & \multicolumn{2}{|c|}{$45(30-59)$} & \multicolumn{2}{|c|}{$49(28-6 I)$} & 0.065 \\
\hline $28-40$ & 15 & 28.8 & 2 & 9.1 & \\
\hline $4|-6|$ & 37 & 71.2 & 20 & 90.9 & \\
\hline T stage & & & & & 0.509 \\
\hline $\mathrm{TI}$ & 2 & 4.0 & 0 & 0 & \\
\hline $\mathrm{T} 2$ & 31 & 62.0 & 11 & 50.0 & \\
\hline T3 & 13 & 26.0 & 8 & 36.4 & \\
\hline $\mathrm{T} 4$ & 4 & 8.0 & 3 & 13.6 & \\
\hline$N$ stage & & & & & 0.350 \\
\hline No & 20 & 38.5 & 9 & 42.9 & \\
\hline NI & 26 & 50.0 & 10 & 47.6 & \\
\hline N2 & 0 & 0 & 1 & 4.8 & \\
\hline N3 & 6 & 11.5 & 1 & 4.8 & \\
\hline Breast subtype & & & & & 0.728 \\
\hline Luminal A & 6 & 11.5 & 1 & 4.5 & \\
\hline Luminal B & 33 & 63.5 & 14 & 63.6 & \\
\hline Triple-negative & 7 & 13.5 & 3 & 13.6 & \\
\hline Her-2 overexpression & 6 & 11.5 & 4 & 18.2 & \\
\hline $\mathrm{PCR}$ & & & & & 0.456 \\
\hline No & 41 & 83.7 & 19 & 90.5 & \\
\hline Yes & 8 & 16.3 & 2 & 9.5 & \\
\hline
\end{tabular}

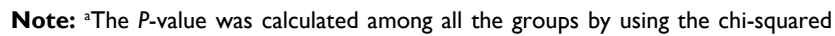
test.

Abbreviation: pCR, pathological complete response.

\section{Serum miR-4530 expression was correlated with the chemotherapeutic sensitivity of NAC patients in the validation phase}

To further investigate the contribution of miR-4530 to the chemotherapeutic response, we conducted qRT-PCR on all samples from both groups. As shown in Figure 1, miR-4530 expression was elevated in the sensitive group compared with that in the resistant group $(P=0.029)$.

ROC curve analysis for the differential expression of miR-4530 between sensitive and resistant patients is depicted in Figure 2. The area under the curve (AUC) for miR-4530 was 0.662 with a standard error of $0.073(P=0.029)$, which indicated a high potential of miR-4530 to be used as a diagnostic biomarker. The sensitivity and specificity were $98.1 \%$ and $86.4 \%$, respectively.

\section{RUNX2 served as a direct target of miR-4530}

Using miRNA structure prediction software such as TargetScan, miRBase Targets, and miRDB, we identified more than 6,079 target genes of miR-4530. Among these,

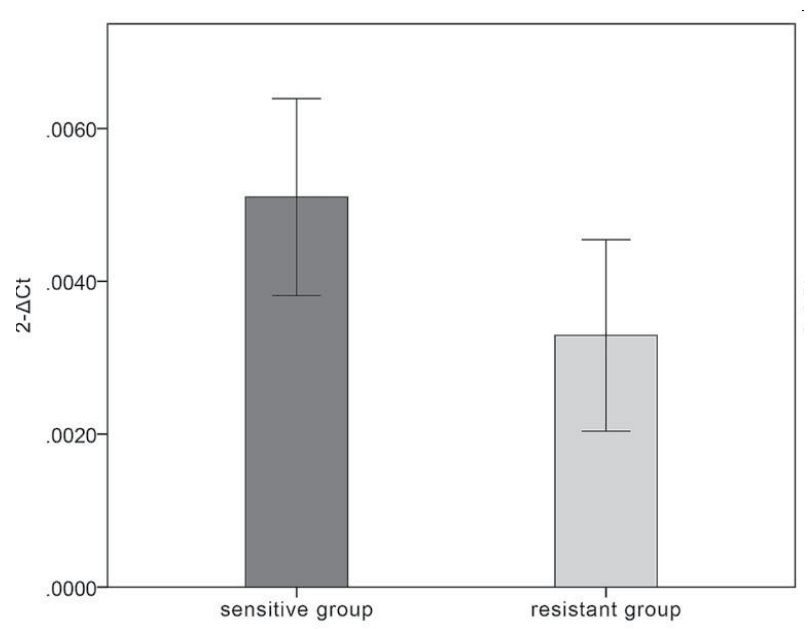

Figure I Different expression levels of miR-4530 between the sensitive and resistant groups were analyzed by quantitative real time-polymerase chain reaction, which were indicated by the columns.

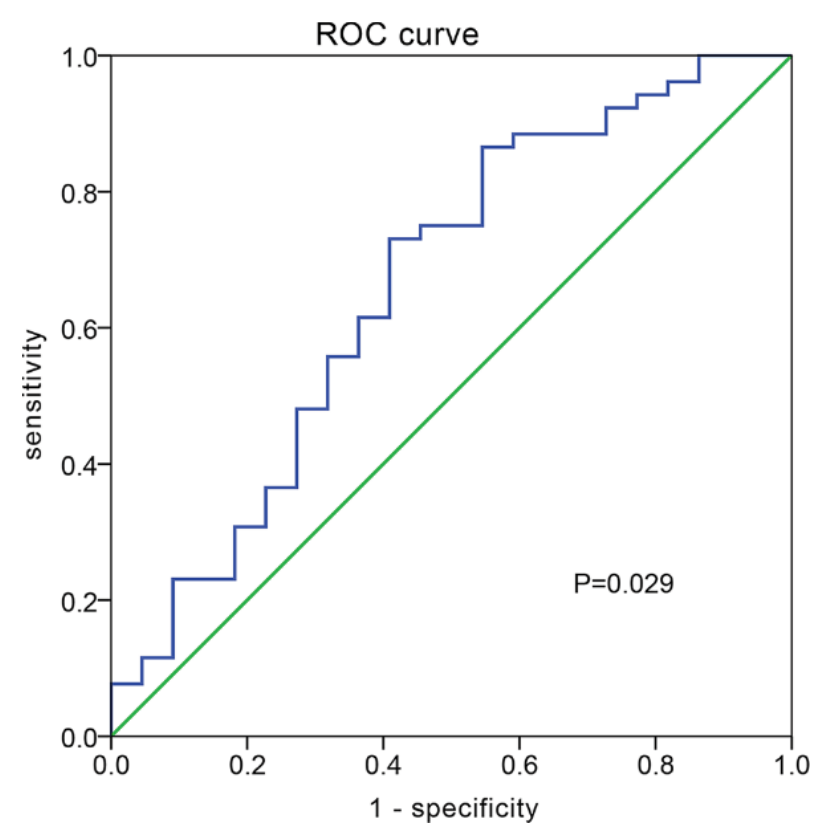

Figure 2 Receiver operating characteristics (ROC) curve analysis for the differential expression of miR-4530 was performed to assess the predictive accuracy.

we identified several genes involved in chemotherapeutic resistance, including RUNX2, Bcl-2, E2F2, PIK3, and Rab.

Further, we examined RUNX2 expression at the transcription level in HS578T breast cancer cell line. Figure 3 illustrates that RUNX2 was expressed at significantly lower levels in HS578T cells, which were co-transfected with the miR-4530 mimics compared with miR-control $(P<0.001)$.

To explore whether RUNX2 was regulated by miR4530 through direct binding to its $3^{\prime}$-UTR, either wild-type or mutant 3 -UTR fragments were inserted into the region immediately downstream of the luciferase reporter con- 


\section{HS578T}

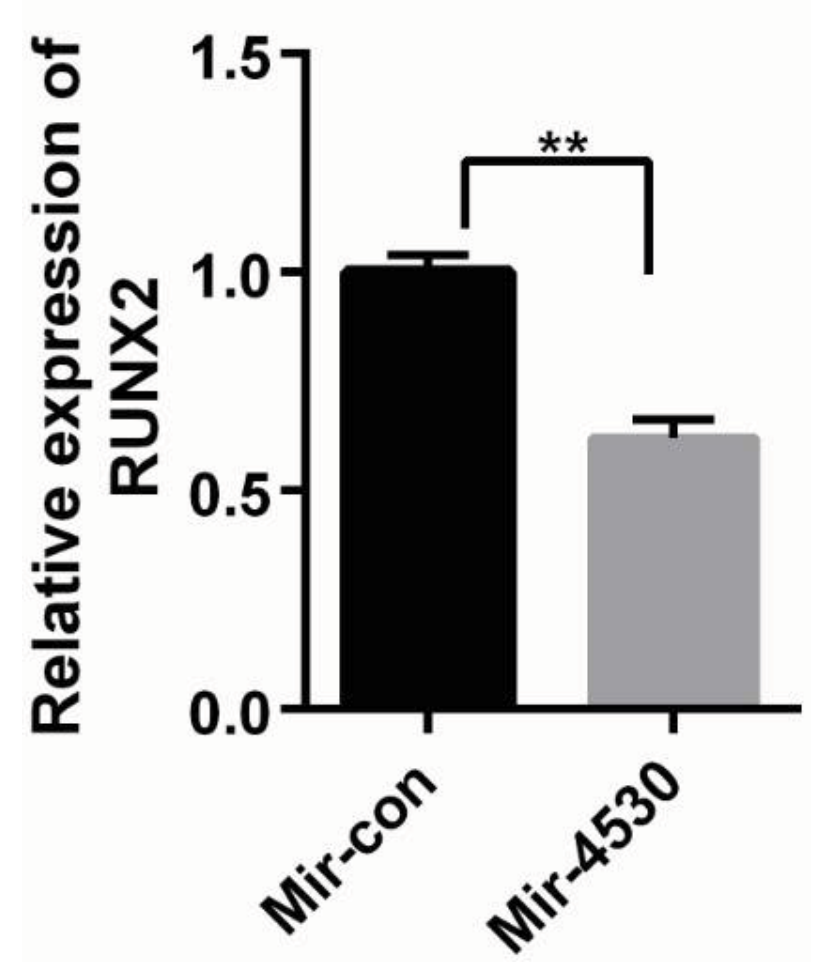

Figure 3 Relative expression of RUNX2 in HS578T cells, which were co-transfected with the miR-4530 mimics or miR-control $(* * P<0.001)$.

Note: RUNX2 levels were examined by reverse transcription PCR.

Abbreviation: RUNX2, Runt-related transcription factor 2.

structs. miR-4530 was then co-transfected with different luciferase 3'-UTR constructs into HEK293T cells. As shown in Figure 4, compared with negative control, the over expression of miR-4530 could significantly reduce the relative RUNX2 3'-UTR luciferase activity in wild-type constructs $(P=0.038)$, not in mutant-type constructs. It indicated that miR-4530 may sensitize breast cancer to NAC by inhibiting activities of RUNX2.

\section{Discussion}

NAC has been used in patients with locally advanced inoperable disease; moreover, NAC has been validated to induce tumor downstaging by shrinking the tumor size, thereby facilitating breast conserving therapy. The identification of reliable biomarkers that may identify patients who would benefit most from chemotherapy is of great importance because breast cancer is a heterogeneous disease. Circulating miRNAs are highly stable and more easily detected compared to tissue-based biomarkers, which opens up new possibilities in predicting a patient's chemotherapeutic response. Therefore,

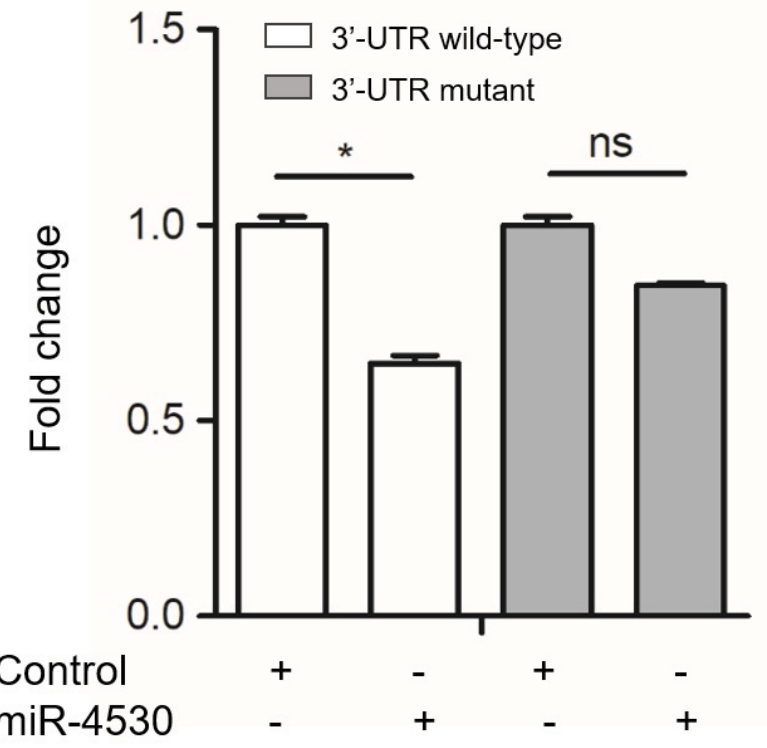

Figure 4 Relative luciferase activities of wild-type or mutant RUNX2 3'-UTRs were determined in HEK293T cells, which were co-transfected with the miR-4530 mimics or negative control.

Note: Firefly luciferase activity was normalized to the internal Renilla luciferase activity $(* P<0.05)$.

Abbreviations: ns, no significance; RUNX2, Runt-related transcription factor 2.

it is urgent for us to focus on serum miRNAs as potential biomarkers for chemotherapy.

There are a growing number of miRNA studies that have focused on the involvement of these micromolecules in chemotherapeutic resistance and the possible distinct mechanisms involved, including drug transport and metabolism, apoptosis, cell cycle regulation, epithelial-mesenchymal transition, DNA methylation, and histone modification. ${ }^{17-20}$ For example, Wang et $\mathrm{al}^{10}$ found that miR-125b overexpression in MCF-7 cells significantly downregulated E2F3 protein expression by binding to a target site in the 3 '-UTR; this could disrupt cell homeostasis because E2F3 plays essential roles in cell proliferation and apoptosis. In our study, we found that elevated expression levels of miR-4530 were observed in NAC-sensitive patients. Using various miRNA structure prediction software such as TargetScan, miRBase Targets, and miRDB, we searched and identified more than 6,079 target genes of miR-4530, some of which are associated with chemotherapeutic resistance, including Bcl-2, E2F2, PIK3, and Rab.

Intriguingly, we also observed that miR-4530 expression was associated with RUNX2, which is one of the best known transcription factors associated with osteoblast differentiation. Its protein structure consists of a RUNX domain, three transcriptional activation regions, a transcriptional inhibition region, and a nuclear localization signal. ${ }^{21,22}$ Recently, it 
has been revealed that RUNX2 has an oncogenic function in the etiology of multiple tumors, including breast cancer. Taipaleenmäki et $\mathrm{al}^{23}$ indicated that reconstitution of RUNX2 in MDA-MB-231-luc cells delivered with miR-135 and miR-203 could impair progression of breast cancer and metastatic bone disease. In addition, elevated RUNX2 levels in breast cancer patients have been shown to correlate with poor clinical outcomes and prognosis, especially estrogen receptor-negative tumors. ${ }^{24}$ Above all, Ozaki et $\mathrm{al}^{25}$ found that RUNX2 interacted with p53 and inhibited p53-dependent apoptosis in response to adriamycin-mediated DNA damage. Furthermore, they demonstrated that forced expression of RUNX2 remarkably decreased the expression of target genes downstream of p53. Instead, p53 promoted irreversible apoptosis through transactivating its pro-apoptotic target genes BAX, NOXA, and PUMA and eliminated cells with severe DNA damage. In accordance with these results, we boldly predict that miR-4530 may sensitize breast cancer to NAC by suppressing RUNX2. However, the detailed mechanism requires further exploration with large-scale functional studies.

Inevitably, our study contains several limitations. First, in terms of sample sizes, this was a retrospective study in a single center with a small cohort of patients receiving NAC; furthermore, the distribution of these patients between the sensitive and resistant groups was imbalanced. Second, we only calculated the relative expression of miR-4530 between the sensitive and resistant groups. Additional studies are necessary to elucidate the detailed functional mechanism responsible for chemotherapeutic drug resistance at protein and cellular levels.

\section{Conclusion}

Our results indicated that elevated serum miR-4530 levels may sensitize breast cancer to taxane- and anthracyclinebased NAC. In addition, we speculate that miR-4530 may be involved in RUNX2-mediated p53-dependent apoptosis, thus offering a potential new biomarker for predicting the chemosensitivity of breast cancer and providing opportunities to create tailored therapies.

\section{Acknowledgment}

This work was supported by grants from the Training Plan of Middle-aged and Young Talents of the Fujian Province Health and Family Planning Commission (2014-ZQNZD-10), the National Natural Science Foundation of China (81672817), and the Wu Jieping Medical Foundation (320.6750.14142).

\section{Disclosure}

The authors report no conflicts of interest in this work.

\section{References}

1. Siegel RL, Miller KD, Jemal A. Cancer statistics, 2015. CA Cancer J Clin. 2015;65(1):5-29.

2. Fisher B, Brown A, Mamounas E, et al. Effect of preoperative chemotherapy on local-regional disease in women with operable breast cancer: findings from National Surgical Adjuvant Breast and Bowel Project B-18. J Clin Oncol. 1997;15(7):2483-2493.

3. Fisher B, Bryant J, Wolmark N, et al. Effect of preoperative chemotherapy on the outcome of women with operable breast cancer. J Clin Oncol. 1998;16(8):2672-2685.

4. Wolmark N, Wang J, Mamounas E, Bryant J, Fisher B. Preoperative chemotherapy in patients with operable breast cancer: nine-year results from National Surgical Adjuvant Breast and Bowel Project B-18. J Natl Cancer Inst Monogr. 2001;(30):96-102.

5. Ambros V. The functions of animal microRNAs. Nature. 2004; 431(7006):350-355.

6. Bartel DP. MicroRNAs: genomics, biogenesis, mechanism, and function. Cell. 2004;116(2):281-297.

7. He L, Hannon GJ. MicroRNAs: small RNAs with a big role in gene regulation. Nat Rev Genet. 2004;5(7):522-531.

8. Jung EJ, Santarpia L, Kim J, et al. Plasma microRNA 210 levels correlate with sensitivity to trastuzumab and tumor presence in breast cancer patients. Cancer. 2012;118(10):2603-2614.

9. Li Q, Liu M, Ma F, et al. Circulating miR-19a and miR-205 in serum may predict the sensitivity of luminal A subtype of breast cancer patients to neoadjuvant chemotherapy with epirubicin plus paclitaxel. PLoS One. 2014;9(8):e104870.

10. Wang H, Tan G, Dong L, et al. Circulating MiR-125b as a marker predicting chemoresistance in breast cancer. PLoS One. 2012;7(4):e34210.

11. Wu X, Somlo G, Yu Y, et al. De novo sequencing of circulating miRNAs identifies novel markers predicting clinical outcome of locally advanced breast cancer. J Transl Med. 2012;10:42.

12. Zhao R, Wu J, Jia W, et al. Plasma miR-221 as a predictive biomarker for chemoresistance in breast cancer patients who previously received neoadjuvant chemotherapy. Onkologie. 2011;34(12):675-680.

13. Mo MH, Chen L, Fu Y, Wang W, Fu SW. Cell-free circulating miRNA biomarkers in cancer. $J$ Cancer. 2012;3:432-448.

14. Turchinovich A, Weiz L, Langheinz A, Burwinkel B. Characterization of extracellular circulating microRNA. Nucleic Acids Res. 2011;39(16):7223-7233.

15. Vickers KC, Palmisano BT, Shoucri BM, Shamburek RD, Remaley AT. MicroRNAs are transported in plasma and delivered to recipient cells by high-density lipoproteins. Nat Cell Biol. 2011;13(4):423-433.

16. Lawrie CH, Gal S, Dunlop HM, et al. Detection of elevated levels of tumour-associated microRNAs in serum of patients with diffuse large B-cell lymphoma. Br J Haematol. 2008;141(5):672-675.

17. Kastl L, Brown I, Schofield AC. miRNA-34a is associated with docetaxel resistance in human breast cancer cells. Breast Cancer Res Treat. 2012;131(2):445-454.

18. Pogribny IP, Filkowski JN, Tryndyak VP, Golubov A, Shpyleva SI, Kovalchuk O. Alterations of microRNAs and their targets are associated with acquired resistance of MCF-7 breast cancer cells to cisplatin. Int J Cancer. 2010;127(8):1785-1794.

19. Yang G, Wu D, Zhu J, et al. Upregulation of miR-195 increases the sensitivity of breast cancer cells to Adriamycin treatment through inhibition of Raf-1. Oncol Rep. 2013;30(2):877-889.

20. Yang Q, Wang Y, Lu X, et al. MiR-125b regulates epithelial-mesenchymal transition via targeting Sema4C in paclitaxel-resistant breast cancer cells. Oncotarget. 2015;6(5):3268-3279.

21. Kojima H, Uemura T. Strong and rapid induction of osteoblast differentiation by Cbfa1/Til-1 overexpression for bone regeneration. $J$ Biol Chem. 2005;280(4):2944-2953. 
22. Komori T. Regulation of skeletal development by the Runx family of transcription factors. J Cell Biochem. 2005;95(3):445-453.

23. Taipaleenmäki H, Browne G, Akech J, et al. Targeting of Runx 2 by miR135 and miR-203 impairs progression of breast cancer and metastatic bone disease. Cancer Res. 2015;75(7):1433-1444.

24. Onodera Y, Miki Y, Suzuki T, et al. Runx2 in human breast carcinoma: its potential roles in cancer progression. Cancer Sci. 2010;101(12):2670-2675.
25. Ozaki T, Wu D, Sugimoto H, Nagase H, Nakagawara A. Runt-related transcription factor 2 (RUNX2) inhibits p53-dependent apoptosis through the collaboration with HDAC6 in response to DNA damage. Cell Death Dis. 2013;4:e610. 


\section{Supplementary material}

Table S I Differentially expressed miRNAs between the sensitive and resistant groups

\begin{tabular}{lll}
\hline Systematic name & P-value & Fold change \\
\hline hsa-miR-4746-3p & 0.012 & 4.940962 \\
hsa-miR-6745 & 0.007163 & 4.190171 \\
hsa-miR-550a-3-5p & 0.042795 & 3.624352 \\
hsa-miR-4472 & 0.04346 & 3.297555 \\
hsa-miR-3648 & 0.046596 & 2.558925 \\
hsa-miR-4530 & 0.027919 & 2.163538 \\
hsa-miR-4689 & 0.032995 & 2.072619 \\
hsa-miR-8072 & 0.047711 & 2.06716 \\
hsa-miR-135a-3p & 0.047406 & 2.025247 \\
hsa-miR-572 & 0.047783 & 1.952809 \\
hsa-miR-3960 & 0.029935 & 1.800699 \\
hsa-miR-483-5p & 0.017774 & 1.744576 \\
hsa-miR-3667-5p & 0.026681 & 1.517467 \\
hsa-miR-6728-5p & 0.050413 & 1.470332 \\
hsa-miR-4669 & 0.046815 & 1.463033 \\
\hline
\end{tabular}

\section{Publish your work in this journal}

Cancer Management and Research is an international, peer-reviewed open access journal focusing on cancer research and the optimal use of preventative and integrated treatment interventions to achieve improved outcomes, enhanced survival and quality of life for the cancer patient. The manuscript management system is completely online and includes a very quick and fair peer-review system, which is all easy to use. Visit $\mathrm{http}: / / \mathrm{www}$.dovepress.com/testimonials.php to read real quotes from published authors. 